\title{
Use of the HCR-20 for violence risk assessment: views of clinicians working in a secure inpatient mental health setting
}

Geoffrey L. Dickens and Laura E. O'Shea

This is the author accepted manuscript, ( $९ 2016$ Emerald Publishing Limited. Emerald does not grant permission for this article to be further copied/distributed or hosted elsewhere without the express permission from Emerald Publishing Limited.

Available from: http://dx.doi.org/10.1108/JFP-08-2016-0039 


\section{Use of the HCR-20 for violence risk assessment: views of clinicians working in a secure inpatient mental health setting}

Geoffrey L Dickens* Professor of Mental Health Nursing, Division of Mental Health Nursing and Counselling, Abertay University, Bell Street Dundee. DD1 1GH. T: 07914157365 E: g.dickens@abertay.ac.uk

Laura E. O’Shea Research assistant, St Andrew’s Healthcare, Northampton. NN1 5DG.

*Corresponding author 


\begin{abstract}
Purpose: To explore how raters combine constituent components of HCR-20 risk assessment for inpatient aggression, and how relevant they rate the tool for different diagnostic and demographic groups.

Design/methodology/approach: A cross-sectional survey design was used. $N=45$ mental health clinicians working in a secure hospital responded to an online survey about their risk assessment practice.
\end{abstract}

Findings: HCR-20 Historical and Clinical sub-scales were rated the most relevant to violence prediction but four of the five items rated most relevant were Historical items. A recent history of violence was rated more important for risk formulation than Historical and Risk management items, but not more important than Clinical items. While almost all respondents believed predictive accuracy would differ by gender, the tool was rated similarly in terms of its relevance for their client group by people working with men and women respectively.

Research limitations/ implications: This was an exploratory survey and results should be verified using larger samples.

Practical implications: Clinicians judge recent violence and Clinical items most important in inpatient violence risk assessment but may over-value historical factors. They believe that recent violent behaviour is important in risk formulation; however, while recent violence is an important predictor of future violence, the role it should play in SPJ schemes is poorly codified.

Social implications: It is important that risk assessment is accurate in order to both protect the public and to protect patients from overly lengthy and restrictive detention.

Originality/ value: Despite the vast number of studies examining the predictive validity of tools like HCR-20 very little research has examined the actual processes and decision-making behind formulation in clinical practice. 


\section{Background}

Structured professional judgement (SPJ) approaches are recommended for the assessment of risk for violence among criminal justice and mental health populations (National Institute for Mental Health in England, 2004). SPJ tools, including the Historical Clinical Risk management - 20 (HCR-20; Webster, Douglas, Eaves, \& Hart, 1997) and the Short Term Assessment of Risk and Treatability (START; Webster, Martin, Brink, Nicholls, \& Desmarais, 2009), include schedules of empirically grounded risk factors whose presence, partial presence, or absence raters are required to determine.

SPJ tools differ from actuarial schemes in which a numerical score is calculated, compared with data from validation samples, and used to determine a non-discretionary risk-level assignation according to predetermined cut-off scores. In contrast, SPJ relies on an idiographic reading of the individual patient's risk factor assessment, together with other relevant considerations, before a final risk level (usually high, medium or low) is assigned at the raters' discretion and incorporating their own clinical expertise. While SPJ tools can be used actuarially for research purposes by summing risk schedule items this is not recommended for clinical practice (Webster et al., 1997) and cut-off scores are not provided since the total number of present risk factors is not considered to be the sole determinant of risk (Hart \& Logan, 2011). No detailed exposition of the weight or content that should be placed on various components of the SPJ assessment is provided; indeed, overly directive advice would be contrary to the spirit of the SPJ approach (Hart \& Logan, 2011).

Nevertheless, SPJ tool developers have become increasingly expansive about the processraters should follow when formulating risk estimates. For example, while somewhat rudimentary advice was offered in the manual for HCR-20 version 2 (Webster et al., 1997), developments in version 3 (Douglas, Hart, Webster, \& Belfrage, 2013) are more detailed. A 
seven-step procedure recommended: i) information gathering; ii) establishing presence of risk factors; iii) determining relevance of risk factors; iv) undertaking risk formulation; v) scenario planning; and vi) developing a management plan; vii) finally, the overall summary judgement is made using the information gathered and considerations made during stages 1 to 6. Studies of the predictive efficacy of the HCR-20 for inpatient aggression show that, while a score calculated actuarially is significantly predictive of violence, it fares less well than the overall final judgement (O'Shea, Mitchell, Picchioni, \& Dickens, 2013). Similarly, the START violence risk estimate also has incremental predictive validity over the risk scale score (O'Shea, Picchioni, \& Dickens, 2016). This suggests that the process of formulation described above and/or other elements of arriving at the overall final judgement adds predictive value and, accordingly, examination of these phenomena has the potential to inform risk assessment in practice.

Despite the potential value of understanding clinicians' SPJ ratings-related practice, little is known empirically about how they arrive at their final risk judgement. While trained raters will be aware of the recommended procedure of arriving at a final judgement, it is not known whether this is adhered to; indeed the developers of HCR-20 version 3 acknowledge that "not all steps will be done by all evaluators or in all cases” (Douglas, Hart, Webster, Belfrage, Guy, \& Wilson, 2014, p. 100). Additionally, since SPJ schemes explicitly value the raters’ individual expertise, it is self-evident that their views of different elements of the assessment will influence their rating for good or bad. We recently conducted a study (O’Shea \& Dickens, 2016) to explore the contribution of risk assessment components to summary judgements, and of the contribution of those components and summary judgements, to the prediction of risk outcomes using clinically generated risk assessment data from the START. We found that raters' violence risk estimate was predicted by two START components: lifetime history of violence, and the vulnerabilities scale score (i.e., summed presence of 
items included on the tool's risk schedule). However, the best predictor of actual violent outcomes, with incremental predictive ability above the START components, was our selfdeveloped heuristic indicator representing the presence of violence in the 3-month period prior to assessment. This risk factor is not identified in the START manual for consideration. We believe that, in this instance, raters were over-estimating the importance of risk factors contained in the START vulnerabilities scale, and the lifetime history of violence, in the prediction of short-term risk for violence. If this is the case then a rating of increased risk in the absence of recent history of risk, and the subsequent enactment of restrictive management procedures based on that rating, may be unjustified and thus unethical. This provides further impetus to examine how clinicians formulate risk estimates in practice since they may not be using all the available information in an optimal manner. A further reason for studying how clinicians make risk estimates, and the processes behind those decisions, lies in the relevance or otherwise of the tool and its' components to the individual case at hand. For example, we have previously ascertained that the HCR-20 does not perform equally across demographic and clinical groups (O'Shea, Picchioni, Mason, Sugarman, \& Dickens, 2014) for prediction of inpatient aggression, but the extent to which assessors believe that a tool is relevant to their clinical population when it differs significantly from the validation population is not known. Since we were collecting data from different professional groups we also examined whether professional background was associated with any systematic differences in terms of perceptions of elements of the HCR-20. We have conducted an exploratory survey study of clinicians' views of the HCR-20 in order to explore these issues. The specific aims were to:

- Ascertain which elements of the HCR-20 clinicians believe are most important in risk assessment for inpatient violence and compare this to findings from previous studies of predictive accuracy. 
- Determine whether rating clinicians believe that HCR-20 is equally valid for different populations, and whether they believe it is valid for the population(s) with which they apply the tool in practice.

- Determine whether professional groups differ in terms of their perceptions of performance of elements of the HCR-20.

- Ascertain whether clinicians believe there are risk factors for violence in their clinical populations that are not covered in the HCR-20 schedule.

\section{Method}

\section{Setting and participants}

The study was conducted across four geographical sites of St Andrew's, a charitable provider of specialist secure inpatient mental health care in England. Potential participants were any clinicians with experience of using the HCR-20 for risk assessment. We did not conduct a power calculation to inform desirable sample size since i) the study was exploratory, and ii) no previous study has used these measures so there was insufficient information to estimate power. We aimed for a sample of $N=50$.

\section{Design}

A cross-sectional survey design utilising repeated measures (ratings of clinicians' perceptions of the value of each of the HCR-20's subscales; and ratings of HCR-20 accuracy for different demographic and clinically defined groups) and independent group measures (clinicians' ratings of HCR-20 accuracy for their own patient group versus ratings of clinicians working with other patient groups; ratings of HCR-20 accuracy by professional group). Additionally, participants were surveyed about their perceptions of the most and least important HCR-20 items, and about whether there were risk factors present in their patient group which were not 
addressed by the HCR-20.

\section{Procedure}

The study was approved by the Hospital quality assurance team. A questionnaire was hosted on the organisation's intranet site and a link was circulated via email to all qualified clinicians informing them of the purpose of the study and inviting participation. The survey was available for completion for two weeks and participation was anonymous.

\section{Measures}

We devised a questionnaire comprising items relevant to the study aims (available on request from the authors). In addition to items to ascertain the demographic characteristics of participants, we asked about the patient group that they worked with in terms of gender and diagnostic category (Schizophrenia, Personality disorder, Intellectual disability, Organic/ABI, Developmental). Questions about their use of the HCR-20 related to the overall accuracy of the tool with their patient group (measured on a scale of 1 to 10); the importance of the individual H (Historical), C (Clinical), and R (Risk management) scales in generating an overall risk estimate; and the importance of a lifetime history of violence and of recent (e.g., past 3-month) violence in formulating an overall risk estimate. We also asked participants to indicate whether they believed the HCR-20 was likely to be a more, less, or similarly accurate predictor of violence for gender and diagnostic groups other than those with which they currently worked. Finally, we asked participants to select the five most and five least important items in the prediction of violence with their client group. All our questions related to version 2 of the HCR-20 (Webster et al., 1997) since that was the version in use in the study setting at the time. The HCR-20 version 2 comprises 20 items in three subscales: Historical, Clinical, and Risk management (see Table 1). The questionnaire was circulated to a multidisciplinary reference group for comment; feedback was gathered and appropriate 
amendments made.

>>Insert Table 1 about here $<<$

\section{Analysis}

Data were analysed using SPSS Version 22 (IBM SPSS Statistics, 2013). Sample characteristics, rating of utility of HCR-20 in non-validation samples, and presence of other risk factors for violence were subject to descriptive analyses; comparison of the relative importance of elements of HCR-20 assessment was made using one-way ANOVA; similarly, significant differences between overall HCR-20 accuracy by staff groups working with different groups of patients were made using one way ANOVAs. For analysis of the perceptions of different professional groups we collapsed data to two groups (psychiatrists $n=24$ and all others $n=21$ ) and analysed using independent samples t-tests or non-parametric equivalents where appropriate.

\section{Results}

In total, $N=45$ clinicians completed the survey; characteristics of the sample are presented in Table 2. In addition, psychiatrists had similar total years experience than all other clinicians ( $M=6.24 \pm 9.46$, vs. $M=8.74 \pm 7.42, P=.95)$ but reported more years HCR-20 experience (Median=5+ years vs. Median=2-5 years, $\mathrm{Z}=-3.99, P<.001)$.

\section{>> Insert Table 2 about here $<<$}

\section{Most relevant HCR-20 subscales}

When asked which HCR-20 subscales were of most relevance to risk assessment with their patients the Historical and Clinical subscales were selected as first choice by $n=19$ respondents each (42\%) with the remainder $(n=7 ; 18 \%)$ favouring the risk management 
items. Similarly, almost $80 \%$ also believed that either Historical or Clinical items were the second most relevant suite of items, whilst $n=37$ (82\%) believed risk management items to be the second or third most relevant. In contrast, when we asked respondents to name the five individual HCR-20 items they believed to be most relevant to violence risk assessment, Historical items were the clear leaders; four of the five most frequently listed items were from the Historical scale ('early maladjustment', 'previous violence', 'major mental illness', 'young age at first violence'). Of the dynamic clinical and risk management items only the clinical item 'active symptoms of major mental illness' was in the five most relevant items. Of the individual items rated among the five the least important, three were from the Historical scale ('psychopathy', 'employment problems', and 'personality disorder'), while one item each were drawn from the Clinical ('lack of insight') and Risk management ('noncompliance with remediation attempts') scales.

One-way ANOVA indicated a statistically significant difference between participants’ ratings of the importance of various aspects of the HCR-20 assessment in arriving at a risk estimate (F $[4,219]=3.798, P<.01)$. A Tukey post hoc test revealed that a recent history of violence was rated as more important $(8.36 \pm 1.00)$ than HCR-20 H items $(7.22 \pm 2.10, P<.05)$ and $\mathrm{R}$ items (7.16 $\pm 1.96, P<.01)$, but not $C$ items $(7.53 \pm 1.89)$ or lifetime history of violence (7.87 $\pm 1.27)$. No other significant differences between ratings were found.

\section{HCR-20 as a violence predictor for different diagnostic and demographic groups}

Overall, no respondent believed that the HCR-20 was likely to be a better predictor of inpatient aggression for diagnostic groups that differed from those mostly used in validation samples (see Table 3). For violence prediction among people with intellectual disability or developmental conditions most respondents thought the HCR-20 was likely to be a similar or poorer performer, while a majority were unsure about how it might perform for people with 
brain injury or organic conditions. Interestingly, a more polarised response was evident for gender: one third of respondents thought HCR-20 was likely to be a better performer for women, and just under two thirds a poorer performer; only one respondent estimated similar performance. Of those believing it to be a better performer for women, six (40\%) worked with women, eight (53\%) with men, and one (7\%) with both.

Despite clear differences in beliefs about the predictive ability of the HCR-20 in the whole sample we found no significant differences in ratings of the accuracy of the HCR-20 when we asked respondents about use of the tool with their own patient group. One-way ANOVA revealed that the $M(S D)$ rating for those who worked with men was $5.92( \pm 2.02)$; women $6.29( \pm 1.54)$; both $6.50( \pm 1.23)$; $(\mathrm{F}(2,42)=.347, P=.201) . M(S D)$ for those who worked primarily with those with schizophrenia was $5.75( \pm 1.96)$; PD $6.50( \pm 1.51)$; ID $5.82( \pm$ 2.23); Organic 5.00 ( \pm 2.83); Developmental $6.75( \pm .71),(\mathrm{F}(4,40)=.783, P=.583)$.

\section{>> Insert Table 3 about here $<<$}

\section{Differences in perceptions of the HCR-20 by professional group}

Psychiatrists were not different from other professionals in their ratings of the accuracy of HCR-20 for predicting aggression in their own patient group $(M=6.28 \pm 1.71$ vs. $M=6.08 \pm$ 1.84, $\mathrm{t}=.349, \mathrm{df}=40, P=.63)$. Psychologists and others rated HCR-20 Clinical subscale items as more influential on their overall summary judgement than did psychiatrists $(M=8.28 \pm .96$ vs. $M=6.96 \pm 2.331, \mathrm{t}=2.27, \mathrm{df}=40, P<.05)$; there were no other significant differences on the influence of the Historical or Risk Management items, nor on the influence of any history of violent behaviour or recent aggression. 
Finally, we asked whether respondents felt there were risk factors specific to their clinical population that were not included in the HCR-20. Those working with patients with developmental disabilities provided the most suggestions (changes to the environment, misinterpretation of social norms, communication difficulties/ dysfunction, genetic). Those working with patients with intellectual disability suggested risk factors including overly restrictive practices, inter-patient conflict, lack of structured activity, lack of availability of regular nursing staff, lack of involvement in decision-making, and reliance on pro re nata (as required) medication as a management strategy. Those working with brain injured patients named frontal lobe problems, temporal lobe problems, sensory impairments, and cognitive impairments. Those working with patients with a diagnosis of personality disorder suggested ward atmosphere and ward environment.

\section{Discussion}

While there are numerous studies which demonstrate that the HCR-20 is able to predict inpatient aggression with some accuracy (O'Shea et al., 2013) the current study is one of the few available in the literature to provide information about the assumptions or beliefs held by clinicians who make ratings of risk violence using the tool. Given that rater discretion is integral to the SPJ approach there were number of interesting findings worthy of discussion. First, our finding that different elements of the HCR-20 risk assessment are rated as poorer performers in the prediction of inpatient violence than recent aggression suggests that clinicians’ HCR-20 final judgements might be weighted by their knowledge about the presence or absence of this behaviour. This contrasts with findings from our previous study (O’Shea \& Dickens, 2016) using the START where we found that recent aggression prior to the risk assessment did not predict an elevated summary judgement for violence. However, the same study did suggest that recent aggression was, in fact, the best predictor of actual outcome as opposed to a predictor of the final judgement. If the same were true for the HCR- 
20 then it would suggest that clinicians are correct to rate it as important and, if they do not already, should incorporate a recent aggression heuristic - or re-weight an existing one appropriately - into their summary judgement formulation.

HCR-20 Historical, Clinical, and Risk management items were not rated differently from one another in terms of overall influence on respondents’ final risk judgement. In contrast, respondents identified four Historical scale items in the top five items most likely to be relevant to inpatient aggression. This may superficially appear paradoxical; how can one simultaneously hold the view that historical items are some of the most important determinants of inpatient violence yet not rate them the most important element in the overall judgement? However, it may be that clinicians recognise that the Historical items are relevant to aggression and rate them accordingly when asked this, but do not assign greater relevance to them when formulating overall risk level since, in the current study setting, most patients hold multiple Historical scale risk factors (see e.g., O'Shea et al., 2014). Reliance, therefore, on these items during formulation of overall risk would likely lead them to rate everyone at elevated risk which would not help with prioritisation of resources or identification of risk management interventions. Alternatively, clinicians’ rating of the import of the Historical scale as a whole may not reflect their rating of a subset of individual items. From this perspective it is possible that clinicians really do overvalue the subset of Historical items related to early maladjustment, previous violence, major mental illness, and young age at first violent episode. Such items are relatively static and not amenable to change through intervention. Their contribution in formulating an overall risk judgement is, on the balance ofprobabilities, likely to weight that assessment towards stasis. Importantly, if these are seen as the most important risk factors for aggression it could lead to a lack of focus on more dynamic items which, if successfully addressed, might reduce risk. The HCR-20 version 3 potentially circumvents this concern by requiring raters to determine the relevance of the risk 
as well as the presence.

There were mixed findings regarding the applicability of HCR-20 risk assessment to groups who differ substantially from validation populations. No respondents believed that HCR-20, when used for diagnostic groups including patients with intellectual or developmental disability or organic conditions, outperformed its predictive ability with regard to validation samples; a small minority for each diagnostic condition thought it might perform equally, but most felt the tool was likely perform more poorly or were unsure. This was reflected in respondents working with niche clinical samples naming a number of further risk factors that they believed to be important while those working with patients like those in validation samples reported fewer. However, about a third of respondents, and two fifths of those working with women, thought that the tool might perform better for women than for male only validation samples. This is interesting since a study of differential predictive validity conducted in the current study setting (O'Shea et al., 2014) demonstrated that the HCR-20 did indeed have superior efficacy when applied to women compared to men. In fact, while all but one ( $n=44,97.7 \%)$ respondent expressed that the predictive validity of HCR-20 would likely differ between men and women (i.e., would either be better or worse but not the same); ratings of the accuracy of HCR-20 for their own client group by clinicians working with men and women respectively did not differ significantly. Thus, while subjectively clinicians believed that the tool had better predictive ability for one group or the other, clinicians who worked with each group rated predictive ability similarly. We suspect the practical value of this finding is that those using the tool with women should be reassured that perception of it as a relatively inferior performer are possibly misplaced.

There was only one difference between different professional groups: namely that nonpsychiatrists reported that HCR-20 clinical items had significantly more influence on their overall summary judgements than did psychiatrists. This should be interpreted with caution 
since psychiatrists had significantly more years experience and, as a result, this may be a result of experience rather than profession. Nevertheless, it is interesting to find that, potentially, there are significantly different ways of formulating a summary judgement. The research implication is a need to determine whether the perceived import placed on certain aspects leads to a qualitatively or quantitatively different summary judgement; if there is, then there is an additional need to investigate whether one method provides a judgement with greater predictive accuracy.

\section{Limitations}

This was a small survey conducted across one provider of specialist secure mental health services, including many participants who work with patients for whom the HCR-20 was not specifically developed but is used due to a lack of alternative tools. Findings therefore may not be generalizable to more mainstream forensic, secure services. The questionnaire was developed to answer study aims and did not possess known psychometric properties. Participants were self-selecting and may not have been representative of the clinical workforce.

\section{Conclusions}

In practice clinicians may overvalue some HCR-20 historical items when conducting violence risk assessment. They appear to appreciate the role that recent violence plays in inpatient risk though this is not a formal part of the HCR-20 assessment. Our results suggest that clinicians lack confidence in the ability of the HCR-20 to be of optimal value for assessment of those with diagnostic conditions outwith its validation samples. As a result, we recommend that more development is required in terms of risk assessment for people with disorders other than schizophrenia or personality disorder. The picture in terms of its value from a gendered perspective is more complex since the perception of inferior performance 
with women may be misplaced. There is, therefore, a need to test tools developed for women, such as the Female Additional Manual (de Vogel, de Vries Robbé, van Kalmthout, \& Place, 2014), to ensure they really do add value.

\section{Implications for practice}

- Clinicians using HCR-20 for violence risk assessment in secure forensic mental health settings should remain mindful of, and periodically question, their own assumptions and beliefs about the relative value of aspects of HCR-20 assessment.

- Those working with people with mental health diagnoses that are significantly different from HCR-20 validation samples appear knowledgeable about the relative value of the tool with those patients and should be supported to supplement assessment with information from more appropriate tools where viable.

- Those working with women should be aware that their belief in the relatively poor performance of HCR-20 for that group is not supported either in evidence about actual performance, nor in comparative ratings of accuracy for men when compared with clinicians who work with men in the current study. 


\section{References}

de Vogel, V., de Vries Robbé, M., van Kalmthout, W., Place, C. (2014). Female Additional Manual (FAM): Additional guidelines to the HCR-20V3 for assessing risk for violence in women. Utrecht, Netherland: Van der Hoeven Kliniek. Retrieved from http://www.violencebywomen.com/_files/FAM\%20to\%20be\%20used\%20with\%20HCR20\%20Version\%203\%20-\%20English\%20version\%202014.pdf

Douglas, K.S., Hart, S.D., Webster, C.D., Belfrage, H. (2013). HCR-20V3: Assessing risk of violence - User guide. Burnaby, Canada: Mental Health, Law, and Policy Institute, Simon Fraser University.

Douglas, K.S., Hart, S.D., Webster, C.D., Belfrage, H., Guy, L.S., Wilson, C.M. (2014). Historical-Clinical-Risk Management-20, Version 3 (HCR-20 ${ }^{\mathrm{V} 3}$ ): Development and overview. International Journal of Forensic Mental Health, 13, 93-108.

Hart, S.D. \& Logan, C., 2011. Formulation of violence risk using evidence-based assessments: the structured professional judgment approach. In: P. Sturmey, P. \& M. McMurran (Eds.), Forensic case formulation. Chichester, UK: Wiley-Blackwell.

National Institute for Mental Health in England (2004). Mental health policy implementation guide. London: Department of Health. Retrieved from: http://www.teamteachtutors.co.uk/guidance/documents/NIMHE\%20guidance\%20feb04.pdf

O’Shea, L.E. \& Dickens, G.L. (2016) Role of assessment components and recent adverse outcomes in risk estimation and prediction: Use of the Short Term Assessment of Risk and Treatability (START) in an adult secure inpatient mental health service. Psychiatry Research, 240, 398-405. DOI: 10.1016/j.psychres.2016.04.068

O'Shea, L.E., Mitchell, A.E., Picchioni, M.M., \& Dickens, G.L. (2013). Moderators of the predictive efficacy of the Historical, Clinical and Risk Management-20 for aggression in psychiatric facilities: Systematic review and meta-analysis. Aggression and Violent Behavior $18,255-270$.

O’Shea, L.E., Picchioni, M.M. and Dickens, G.L. (2016) The predictive validity of the ShortTerm Assessment of Risk and Treatability (START) for multiple adverse outcomes in a secure psychiatric inpatient setting. Assessment, 23, 150-162. doi:

$10.1177 / 1073191115573301$

O'Shea, L.E., Picchioni, M.M., Mason, F.L., Sugarman, P.A., \& Dickens, G.L. (2014). Differential predictive validity of the Historical, Clinical and Risk Management Scales (HCR-20) for inpatient aggression. Psychiatry Research, 220, 669-678. doi: 10.1016/j.psychres.2014.07.080

Webster, C.D., Martin, M.L., Brink, J., Nicholls, T.L., Desmarais, S.L. (2009). Manual for the Short-Term Assessment of Risk and Treatability (START) (Version 1.1). Coquitlam, Canada: British Columbia Mental Health and Addiction Services, 
Webster, C.D., Douglas, K.S., Eaves, D. \& Hart, S.D., 1997. HCR-20: Assessing risk for violence (version 2). Mental Health, Law and Policy Institute, Simon Fraser University, Burnaby, British Columbia. 
Table 1: HCR-20 version 2 items

\begin{tabular}{lll}
\hline Historical items & Clinical items & Risk management items \\
\hline $\begin{array}{l}\text { H1 Previous violence } \\
\text { H2 Young age at first } \\
\text { incident }\end{array}$ & C1 Lack of insight & R1 Plans lack feasibility \\
$\begin{array}{l}\text { H3 Relationship } \\
\text { instability }\end{array}$ & $\begin{array}{l}\text { C2 Negative attitudes } \\
\text { C3 Active symptoms of major }\end{array}$ & R2 Exposure to destabilizers \\
$\begin{array}{l}\text { H4 Employment } \\
\text { problems }\end{array}$ & C4 Impulsivity & R3 Lack of personal support \\
H5 Substance use & & $\begin{array}{l}\text { R4 Noncompliance with } \\
\text { remediation attempts }\end{array}$ \\
$\begin{array}{l}\text { problems } \\
\text { H6 Major mental } \\
\begin{array}{l}\text { illness } \\
\text { H7 Psychopathy }\end{array}\end{array}$ & C5 Unresponsive to treatment \\
$\begin{array}{l}\text { H8 Early } \\
\text { maladjustment }\end{array}$ & R5 Stress \\
$\begin{array}{l}\text { H9 Personality disorder } \\
\text { H10 Prior supervision } \\
\text { failure }\end{array}$ & \\
\hline
\end{tabular}


Table 2: Sample characteristics $(N=45)$

\begin{tabular}{ll}
\hline & n (\%) \\
\hline Profession: & $24(53.3)$ \\
Psychiatrist & $18(40)$ \\
Psychologist & $1(2.2)$ \\
Nurse & $2(4.4)$ \\
Other & \\
Patient group gender: & $25(55.6)$ \\
Men & $14(31.1$ \\
Women & $6(13.3)$ \\
Both & \\
Patient group diagnoses: & $11(24.4)$ \\
Schizophrenia & $9(20.0)$ \\
Personality disorder & $4(8.9)$ \\
Intellectual disability & $2(4.4)$ \\
Organic/ABI & $8(17.8)$ \\
Developmental & $11(24.4)$ \\
$2+$ categories & \\
Years experience: & $1(2.2)$ \\
$<1$ & $14(31.1)$ \\
1 to 2 & $12(26.7)$ \\
3 to 5 & $17(37.8)$ \\
$>5$ & $1(2.2)$ \\
Missing & \\
Years HCR-20 experience: & \\
$<1$ & $5(11.1)$ \\
1 to 2 & $6(13.3)$ \\
3 to 5 & $13(28.9)$ \\
$>5$ & $21(46.7)$ \\
\hline
\end{tabular}


Table 3: Estimated value of HCR-20 for prediction of inpatient aggression by diagnosis/gender relative to validation samples of males with schizophrenia personality disorder diagnoses

\begin{tabular}{cllll}
$\begin{array}{l}\text { HCR-20 for people } \\
\text { with... }\end{array}$ & $\begin{array}{l}\text { ID } \\
n(\%)\end{array}$ & $\begin{array}{l}\text { Organic/ABI } \\
n(\%)\end{array}$ & $\begin{array}{l}\text { Developmental } \\
n(\%)\end{array}$ & $\begin{array}{l}\text { Women } \\
n(\%)\end{array}$ \\
\hline Diagnoses is... & & & & $15(33.3)$ \\
Better & $0(0)$ & $0(0)$ & $0(0)$ & $1(2.2)$ \\
Same & $15(33.3)$ & $6(13.3)$ & $9(20.0)$ & $29(64.4)$ \\
Worse & $12(26.7)$ & $12(26.7)$ & $15(33.3)$ & $0(0)$ \\
Unsure & $16(35.6)$ & $27(60.0)$ & $17(37.8)$ & $0(0)$ \\
Missing & $2(4.4)$ & $0(0)$ & $3(6.7)$ & \\
... than for validation samples with schizophrenia and/or personality disorder &
\end{tabular}

